\section{LONG-COURSE, BUT NOT SHORT-COURSE, DEXAMETHASONE TREATMENT IN ELBW INFANTS RESULTS IN HIGHER INTELLECTUAL IMPAIRMENT RATES AT PRESCHOOL AGE}

\author{
M.D. Ahronovich ${ }^{1,2}$, K. Erickson², I.S. Baron ${ }^{1}$, \\ R. Baker ${ }^{1,2}$, F.R. Litman ${ }^{1,2}$ \\ ${ }^{1}$ Pediatrics, Inova Fairfax Hospital for Children, \\ ${ }^{2}$ Neonatology, Fairfax Neonatal Associates at \\ Inova Fairfax Hospital for Children, \\ Falls Church, VA, USA
}

Background and aims: Controversy exists regarding dexamethasone treatment in preterm infants with respiratory failure. We assessed the relationship between dexamethasone (DEX) exposure and length of treatment with intellectual outcome in extremely low birth weight newborns (< 1000 g; ELBW).

Design/methods: We studied 57 ELBW and 90 term-born children at age 3 (2004-2006 births). There were three groups of ELBW participants: NODEX (untreated; $n=38$ ); short-course DEX (116 days; $n=14)$; and long-course DEX (17-42 days; $n=5)$. DEX treatment was limited to use in infants with severe respiratory failure who were $\geq 21$ days of age and $\geq 27$ weeks corrected gestation. General intelligence (Differential Ability Scales-II General Conceptual Ability [GCA]) scores age adjusted for prematurity < 85 were coded as impaired. Chisquare test compared impairment rates.

Results: Impairment rates $(\mathrm{IQ}<85)$ were: $6.7 \%$ for term-born; $13.2 \%$ for NODEX; $14.2 \%$ for short-course; and $60.0 \%$ for long-course (Chisquare=14.46, $p=.002$ ). Post-hoc comparisons indicate NODEX and short-course groups did not differ significantly while long-course resulted in significantly higher impairment rates compared to both NODEX $(p=.01)$ and short-course groups $(p=.002)$.



[Impairment rates and DEX treatment]

Conclusions: We found short-course dexamethasone treatment did not increase the risk of intellectual impairment in ELBW children at age 3. Concerns regarding developmental outcome may be warranted but should not preclude consideration of short-term dexamethasone treatment for severe respiratory failure in ELBW infants. However, treatment extended beyond 16 days significantly increased the risk of intellectual impairment.

431

\section{PREDICTORS OF DECREASED FLUID TOLERANCE IN VLBW INFANTS}

T. Vuohelainen ${ }^{1,2}$, R. Ojala ${ }^{1,2}$, A. Virtanen ${ }^{2}$, T. Luukkaala ${ }^{3}$, M. Janas ${ }^{1}$, P. Korhonen ${ }^{1}$, O. Tammela ${ }^{1,2}$

${ }^{1}$ Department of Paediatrics, Tampere University Hospital, ${ }^{2}$ Paediatric Research Centre, University of Tampere, ${ }^{3}$ Science Center, Pirkanmaa Hospital District, Tampere, Finland

Objective: The goal was to elucidate predictors of decreased fluid tolerance (DFT) in very low birth weight (VLBW) infants. We hypothesized that the fluid retention is linked to severity of pulmonary problems and prolonged respiratory support, especially to nCPAP treatment.

Methods: The investigation was carried out at Tampere University Hospital between years 2001 and 2006. The study population comprised 74 very low birth weight (VLBW) infants born at 29.21 